\title{
« LA SITUATION COLONIALE » CHEZ GEORGES BALANDIER
} Relecture historienne

Isabelle Merle

Armand Colin | «Monde(s)»

$2013 / 2 \mathrm{~N}^{\circ} 4 \mid$ pages 211 à 232

ISSN 2261-6268

ISBN 9782200928629

Article disponible en ligne à l'adresse :

https://www.cairn.info/revue-mondes1-2013-2-page-211.htm

Distribution électronique Cairn.info pour Armand Colin.

(C) Armand Colin. Tous droits réservés pour tous pays.

La reproduction ou représentation de cet article, notamment par photocopie, n'est autorisée que dans les limites des conditions générales d'utilisation du site ou, le cas échéant, des conditions générales de la licence souscrite par votre établissement. Toute autre reproduction ou représentation, en tout ou partie, sous quelque forme et de quelque manière que ce soit, est interdite sauf accord préalable et écrit de l'éditeur, en dehors des cas prévus par la législation en vigueur en France. Il est précisé que son stockage dans une base de données est également interdit. 


\section{"La situation coloniale " chez Georges Balandier Relecture historienne}

Isabelle Merle

Université de la Nouvelle Calédonie (CNEP)

\section{Résumé}

«La notion de situation coloniale», article publié d'abord en 1951 dans les Cahiers internationaux de sociologie puis repris en 1955 et dans les éditions ultérieures de Sociologie actuelle de l'Afrique noire est aujourd'hui très connu. La formule est communément utilisée sans qu'on sache toujours ce qu'elle recouvre. L'enjeu de cette contribution est de revenir sur l'approche socio-historique proposée en son temps par le jeune Balandier, sous l'angle d'une lecture historienne prenant en compte l'étude des contextes coloniaux et leurs effets d'héritage.

Mots-clés : Situation coloniale - Domination Colonisation - Indigènes - Colons.

\section{Abstract}

“The Colonial Situation", in Georges Balandier: an historian's rereading

"The Colonial Situation", a text George Balandier first published in 1951 in the Cahiers internationaux de sociologie, taken up in 1955 and in subsequent editions of Sociology of Black Africa, is well-known today. The objective of this article is to go back over the sociohistorical approach that the young Balandier once put forward, as well as to the content of his text with its objectives and limits from the point of view of a historical reading.

Keywords: Colonial Situation - Colonization Natives - Settlers - Domination.

monde(s), $n^{\circ} 4$, septembre 2013, p. $\underline{211-232}$ 
À l'heure où les études portant sur la colonisation ou les colonisations connaissent, après une longue éclipse, un regain d'intérêt certain, la notion de " situation coloniale » est aujourd'hui communément mobilisée tant par les historiens que par les anthropologues ou les politologues. La formule est parfois utilisée sans référence aucune, comme explicite en elle-même, mais elle est le plus souvent associée à l'article que Georges Balandier publia pour la première fois sous le titre « La situation coloniale. Approche théorique » en 1951 dans les Cahiers internationaux de sociologie.

Ce texte, longtemps oublié, fut remis à l'honneur dans les années 1990 par deux historiens américains, Frederick Cooper, spécialiste de l'Afrique coloniale et Ann Stoler, anthropologue et spécialiste de Sumatra sous domination hollandaise, qui cherchaient alors une nouvelle voie d'approche pour étudier l'objet «colonial». Entre le positivisme d'une histoire coloniale classique et les théorisations des Postcolonial Studies décontextualisantes et anhistoriques, ils proposent, dans l'ouvrage qu'ils publient en 1997 sous le titre Tensions of Empire, un programme de recherche qui revient en quelque sorte aux sources en s'appuyant sur ce texte fondateur de Georges Balandier.

«Si l'effort consiste dans ce volume à souligner le fait que métropole et colonie, colonisateur et colonisé, doivent être nécessairement pensés ensemble dans le cadre d'un nouveau champ analytique et un nouvel agenda de recherche, il attire l'attention sur le fait que cette voie n'est pas nouvelle, d'autres l'ont déjà suivie et bien. En tout premier lieu Georges Balandier (1951) qui de façon convaincante soutenait que la situation coloniale devait être comprise en tant que telle comme une construction culturelle et politique d'un moment particulier avec tous ceux y participant, comme acteurs de l'histoire $»^{1}$.

On remarquera l'usage du terme « construction culturelle et politique » et l'insistance portée aux « moment particulier » et " acteurs de l'histoire ». Ce faisant, les deux auteurs marquent leur ralliement à la posture épistémologique $\mathrm{du}$ jeune Balandier qui voyait dans la situation coloniale un véritable objet sociologique en évolution rapide, exigeant par là-même de le "saisir historiquement $»^{2}$. Ils entendent ainsi défendre, dans le domaine des études coloniales, une approche socio-historique inscrite dans la tradition des sciences sociales contre l'influence alors dominante des Postcolonial Studies, dont Cooper dénonce ironiquement quelques années plus tard la tendance à évacuer « la dimension temporelle, surtout quand on croit repérer des formes de gouvernementalité coloniale au sein d'une "modernité" aux contours mal défi-

1 Frederick Cooper, Ann Stoler, ed., Tensions of Empire. Colonial Culture in a Bourgeois World (Berkeley: University of California Press, 1997), p. 15.

2 Georges Balandier, «La situation coloniale. Approche théorique ", Cahiers internationaux de sociologie, vol. 11, 1951 , p. 44-79, p. 76. 
nis, et qui semble aller de Diderot à Jacques Derrida $»^{3}$.

La notion de « situation coloniale » est très fréquemment mobilisée dans la littérature actuelle en sciences sociales mais rares, au fond, sont les analyses qui ont prêté une attention précise aux conditions de fabrication du texte de Balandier et à son contenu. Un numéro spécial des Cahiers internationaux de sociologie en 2001 semble à la lecture de l'introduction être dédié à la notion de « situation coloniale » mais, outre la réédition partielle de l'article publié en 1951, il ne comporte qu'un seul texte qui lui est véritablement consacré, rédigé par Jean Copans. Élève de Balandier et auteur dans sa jeunesse d'un ouvrage intitulé Anthropologie et impérialisme ${ }^{4}$, ce dernier reprend les références et les apports de l'article, les analysant à l'aune des évolutions de l'anthropologie du développement puis de la mondialisation depuis les années $1950^{5}$. Frederick Cooper ou encore Emmanuelle Sibeud ont, quant à eux, fait un retour au texte en reprenant les

3 Frederick Cooper, Colonialism in Question: Theory, Knowledge, History (Berkeley: University of California Press, 2005); Le colonialisme en question. Théorie, connaissance, histoire, trad. française par Christian Jeanmougin, Paris, Payot, 2010, p. 42.

4 Jean Copans, Anthropologie et impérialisme, Paris, Maspero, 1975.

5 Jean Copans, "La "situation coloniale" de Georges Balandier : notion conjoncturelle ou modèle sociologique et historique ", Cahiers internationaux de sociologie, $n^{\circ} 110,2001$, p. 31-52. idées générales ${ }^{6}$. On notera, l'apport de la toute récente publication de Gregory Mann, consacrée à l'analyse du contexte précis de l'Afrique équatoriale que rencontre Balandier dans les années 1950, et les relations qu'il noue avec les intellectuels, en particulier Mamadou Madeira. Cette récente publication complète fort utilement l'approche qui est proposée ici $^{7}$.

L'enjeu ici est d'y revenir d'une façon plus précise sous l'angle d'un regard d'historien en cherchant d'abord à retracer les étapes de la production d'un écrit qui se fit en trois temps. « La situation coloniale. Approche théorique » fut publié une première fois en 1951 dans les Cahiers internationaux de sociologie ${ }^{8}$. Remanié en 1955, il devint le chapitre pre-

6 Frederick Cooper, Colonialism in Question, op. cit., 2010 (cf. note 3); Emmanuelle Sibeud, Marie-Albane de Suremain, "Histoire coloniale ou Colonial Studies : d'une histoire à l'autre ", in Séverine Awenengo, Pascale Barthélémy, Charles Tshimanga (dir.), Écrire l'histoire de l'Afrique autrement ?, Groupe Afrique noire, cahier $\mathrm{n}^{\circ} 22$, Université Paris VII, L'Harmattan, 2004, p. 73-86.

7 Gregory Mann, "Anticolonialism and Social Science: Georges Balandier, Madeira Keita and 'the Colonial Situation' in French Africa", Comparative Studies in Society and History (2013/1), p. 92-119.

8 Georges Balandier, "La situation coloniale. Approche théorique ", op. cit. (cf. note 2). Balandier proposa en 1952 une première définition de sa «sociologie de la dépendance » au cœur de laquelle il plaçait l'analyse de la « situation coloniale » dans « Contribution à une sociologie de la dépendance ", Cahiers internationaux de sociologie, vol. 12, 1952, p. 47-69. En 1954, il publie "Sociologie de la colonisation et relations entre les sociétés globales ", Cahiers internationaux de sociologie, vol. 17, 1954, p. 1731. 
mier de l'ouvrage Sociologie actuelle de l'Afrique noire sous le titre "La notion de "situation" coloniale ». Ce chapitre fut retouché lors de la réédition du livre en 1963 puis republié à l'identique dans les éditions ultérieures (1971, $1982)^{9}$. On sait que la situation coloniale évolua rapidement entre 1951 et 1963 vers sa propre disparition, devenant alors un objet sociologique déjà dépassé. La question continue pourtant à préoccuper Georges Balandier dans les années 1970. Il y revient dans Anthropologie politique en $1967^{10}$ puis dans Sens et puissance ${ }^{11}$ en 1971, en particulier dans un chapitre intitulé "Sociologie de la colonisation ». Curieusement, ce chapitre rédigé en 1971 revient longuement sur la critique du livre de René Maunier, Sociologie coloniale, publié en 1932 et s'appuie essentiellement sur une bibliographie couvrant les années 1950. Les références apparaissent étrangement datées en 1971, comme si Balandier ne parvenait pas à dépasser les débats et questionnements de l'immédiat aprèsguerre. Ceux-ci ont profondément marqué les hommes de sa génération mais on peut aussi penser que ces débats gardaient vingt ans plus tard une pertinence certaine. Il me semble que c'est encore le cas aujourd'hui à travers l'idée qu'il est important de revenir sur ce moment de rupture que furent ces années 1950

9 Georges Balandier, "La notion de "situation coloniale" ", Sociologie actuelle de l'Afrique noire, Paris, PUF, 1955, 1963, 1971, 1982.

10 Georges Balandier, Anthropologie politique, Paris, PUF, 1967.

11 Georges Balandier, Sens et puissance, Paris, PUF, 1971. au cours desquelles les observateurs bénéficiaient à la fois d'une connaissance précise de la situation coloniale qui leur était contemporaine, et d'une capacité critique qui manquait à leurs prédécesseurs. Au risque de proposer une analyse quelque peu internaliste, cet article vise à chausser les lunettes du jeune Balandier pour ouvrir une sorte de boîte noire : « la situation coloniale ». De quoi s'agit-il précisément ? Comment l'auteur approche-t-il cette réalité sociologique? La richesse de l'analyse de Balandier telle qu'elle est alors formulée, nous intéresse en ce qu'elle peut nous aider à construire aujourd'hui des objets historiques et des problématiques historiennes. «Cinquante ans plus tard [nous dit Cooper] la contribution de Balandier conserve l'actualité d'un travail à la fois engagé et rigoureux ${ }^{12}$. Jean Copans ne dit pas autre chose lorsqu'il conclut ainsi sa « relecture »:

"L'immense majorité de l'humanité vit encore sur le terrain des "situations coloniales" et il n'y a pas de raison d'abandonner une démarche qui fut si productive et qui devrait pouvoir encore rendre de précieux services scientifiques $»^{13}$.

12 Frederick Cooper, Colonialism in Question, op. cit., p. 48 (cf. note 3).

13 Jean Copans, "La "situation coloniale" de Georges Balandier ", op.cit., p. 52 (cf. note 5) 


\section{Les enseignements d'une bibliographie et les trois temps d'un texte}

Comme le signale Jean Copans dans une partie intitulée «Un texte, ses dits, non-dits et lieuxdits ", l'écrit que le jeune Balandier consacre à la situation coloniale "fait d'une certaine manière référence de tout bois ${ }^{14}$ en utilisant une grande diversité de types de textes, les uns tirés d'articles grand public, les autres tirés de la littérature scientifique. Le temps est à l'urgence comme on le sait dans cet après-guerre convaincu de la nécessité de réformer l'empire et la presse générale se fait l'écho de l'intense actualité du moment. D'où le recours à des articles émanant du monde missionnaire, qui reste alors un acteur central du champ colonial ${ }^{15}$, ou à ceux tirés de revues à vocation politique créées dans la foulée de la Libération ${ }^{16}$. Apparaissent aussi l'œuvre d'acteurs de terrain, Robert Delavignette ${ }^{17}$ et Robert Montagne, tous deux anciens administrateurs coloniaux, ou encore les écrits de

14 Ibid., p. 38

15 Louis Joubert, « Le fait colonial et ses prolongements », Le monde non chrétien, $\mathrm{n}^{\circ} 15,1950$, revue de missionnaires catholiques. Est cité aussi un texte de Robert Montagne, «Le bilan de l'œuvre européenne au-delà des mers », contribution à la rencontre catholique de la Semaine sociale de Lyon en 1948, qui donne lieu à une publication : Peuples d'outre-mer et civilisation occidentale.

16 La revue Critique créée par Georges Bataille en 1946 ou la revue Renaissance, née en 1943 à Alger pour participer au renouveau de la pensée politique française et qui disparaît en 1946.

17 Robert Delavignette, Les vrais chefs de l'Empire, Paris, Gallimard, 1939 (rééd. 1946). quelques (rares) figures colonisées et contestataires.

Au-delà de ces articles "grand public » et conformément à la « géométrie "ouverte" de la sociologie défendue par Georges Gurvitch $»^{18}$, Georges Balandier mobilise une bibliographie très éclectique, puisant dans un ensemble de disciplines - histoire, géographie, sociologie, psychologie, science politique, économie et anthropologie -, puisant aussi dans des publications en français autant qu'en anglais. Ce choix est révélateur du fait que «la situation coloniale » ne peut être réfléchie qu'à la confluence de plusieurs disciplines, objet d'étude qui interpelle l'ensemble des sciences sociales. Ce choix est révélateur aussi d'un positionnement original de l'auteur parmi les anthropologues français. Georges Balandier est alors un lecteur assidu de l'anthropologie et de la sociologie américaines, ainsi que de l'anthropologie britannique, en particulier de l'école de Malinowski, qu'il discute et conteste.

Balandier publie une première version de son texte en 1951 au contenu encore marqué par les préjugés d'une époque avec lesquels l'auteur tente de prendre ses distances. Notons les références « aux peuples dits attardés, archaïques ou primitifs » en introduction ou encore la référence aux interprétations des historiens : la « fermeture » des civilisations orientales, mal-

18 Jean Copans, "La "situation coloniale" de Georges Balandier », op. cit., p. 36 (cf. note 5). 
gré les apparences d'occidentalisation ; les relations tendues avec l'Islam qui n'abdique pas le sentiment de sa supériorité et maintient une " compétition qui peut être silencieuse, voilée, mais qui demeure toujours le fond du problème »; l'« ouverture » du monde noir qui s'explique par la disponibilité africaine à l'imitation ", un manque de " confiance aux ressources profondes du cru », "l'Afrique, continent colonial par excellence », « la stagnation économique des masses indigènes »; autant de références qui disparaissent des versions ultérieures de 1955 puis 1963. Le vocabulaire s'épure entre 1951 et 1963 des formules « les plus coloniales » et c'est indéniablement l'introduction qui subit entre 1951 et 1955 la refonte la plus complète. La version de 1951 commence par :

"L'un des événements les plus marquants de l'histoire récente de l'humanité est l'expansion, à travers le globe, de la plupart des peuples européens ; elle a entraîné l'assujettissement quand ce ne fut pas la disparition - de la quasitotalité des peuples dits attardés, archaïques ou primitifs $»^{19}$.

Elle insiste sur le poids de « l'action coloniale [qui] est la forme la plus importante, la plus grosse de conséquences prise par cette expansion européenne » touchant le tiers de la surface du globe et sept cent millions d'individus, « la majeure partie des populations n'apparte-

19 Georges Balandier, «La situation coloniale. Approche théorique ", op. cit., 1951, p. 44 (cf. note 2). nant pas à la race blanche ». Les luttes nationalistes en cours sont qualifiées "d'entreprises difficiles de reconquête ", "d'émancipations donnant lieu à des concessions plus ou moins conditionnelles ${ }^{20}$. En 1951, Balandier croit encore à une " phase technicienne de la colonisation » qui ferait suite à « la phase politicoadministrative ».

Mais en 1955, déjà, la perspective change et le texte commence ainsi :

"Le problème colonial, quelles que soient les apparences, continue à s'imposer à notre attention comme l'une des questions majeures sur laquelle ont à se prononcer les spécialistes des sciences sociales $»^{21}$.

Référence est faite à la montée des nationalismes nouveaux (à laquelle s'ajoutent en 1963 « les réactions induites par la décolonisation ») donnant « à ce problème une acuité, et une actualité, qui ne tolèrent pas l'indifférence $»^{22}$. Évitant les considérations générales, Balandier s'en prend directement à l'anthropologie de son temps qui n'a pas su tenir compte de la situation coloniale en tant que conjoncture particulière. Alors que celle-ci tend à disparaître, il insiste sur l'importance de continuer à l'étudier, y compris en 1963, alors que l'étude des sociétés non plus colonisées mais désormais « affectées par la colonisation » ne

20 lbid., p. 44-45.

21 Georges Balandier, «La notion de situation coloniale », op. cit., 1955, p. 3 (cf. note 9).

22 Ibid., 1963, p. 3 (cf.note 9). 
peut s'accomplir sans ce « complexe qualifié de situation coloniale ${ }^{23}$. L'analyse est délicate du fait des violences et tensions liées à la décolonisation, d'où l'attention plus aigue du sociologue « participant de la première de ces sociétés » à un « continuel effort critique le mettant en garde contre les risques d'une observation trop unilatérale ${ }^{24}$. L'actualité de la guerre d'Algérie ou des émancipations africaines, euphémisée en 1955 sous le terme de « crise des colonisations », qui devient en 1963 une préoccupation des «États nouveaux confrontés avec la tâche de la décolonisation », affecte l'observateur et l'objectivité de sa position. D'où l'importance que Balandier accorde à ce qu'il appelle l'approche théorique de la notion de situation coloniale saisie comme une «totalité » sociologique pour permettre le recul nécessaire face un objet alors très brûlant.

L'effort de réécriture entrepris en 1955 consiste surtout à réduire les perspectives marxistes qui sont développées en 1951. La référence à l'ouvrage célèbre de Lénine, L'impérialisme. Stade suprême du capitalisme, ainsi que la référence d'Hegel au rapport maître-serviteur, disparaissent en 1955. L'idée développée par Charles-André Julien des liens entre capitalisme et monopoles coloniaux ou le rapprochement qu'opérait Jean Guitton entre la question sociale et la question coloniale, le rapport métropolecolonie et le rapport capital-travail, ne sont plus que rapidement évoqués en 1955. Si la référence à l'ouvrage de Joseph Staline, Le marxisme et la question nationale et coloniale (1949) est curieusement maintenue dans les éditions ultérieures du texte, la citation qui lui est consacrée en 1951 est supprimée en $1955^{25}$. Comme le sont aussi les développements consacrés aux concentrations commerciales et industrielles ou ceux consacrés à la question des spoliations foncières et de la prolétarisation des indigènes qu'ont pu analyser Jean Dresh ou Robert Delavignette. Sur un certain nombre de ces points, la version de 1951 laisse entrevoir plus que les versions ultérieures, toute la richesse des travaux entrepris par les historiens et géographes marxistes du temps qui mériteraient aujourd'hui d'être relus attentivement.

Le jeune Balandier en 1955 veut insister sur les aspects politico-administratifs de la colonisation en soulignant l'importance de l'implication des chefs indigènes dans cet appareil de domination et la « dénaturation politique » des sociétés, comme en témoignent ses propres enquêtes menées en pays Fang et Ba-Kongo (Afrique centrale) ou celles que mène alors Meyer Fortes sur la Gold Coast. Il met en valeur les derniers ouvrages de Malinowski Methods of Study of Culture Contact in Africa (1938) et The Dynamics of Culture Change (1945) en s'appuyant en particulier sur la critique radicale qu'en fait Max

25 Georges Balandier, "La situation coloniale. Approche théorique », op. cit., 1951, p. 54 (cf.note 2). 
Gluckman en $1947^{26}$. Il signale aussi de façon plus approfondie les travaux portant sur les rapports raciaux et la psychologie au sens « colonisation de l'esprit », en évoquant des auteurs français qui déjà datent, Saussure, ou Hardy ${ }^{27}$ ou les travaux récents de Bastide et Mannoni, mais aussi Firth, Hallowell, qui tous évoquent ce que Isaac Shapera appelait les «conflits mentaux » résultant du « développement trop rapide de l'individualisme » ou les névroses ou psychoses liées aux changements de modes de vie. Curieusement, même dans les versions de 1963 et ultérieures, Balandier ignore certains travaux qui feront date. L'ouvrage d'Albert Memmi, Portrait du colonisé précédé du Portrait $d u$ colonisateur, préfacé par Jean-Paul Sartre et publié en $1957^{28}$, décrit en détail et d'une façon très innovante l'aliénation psychologique que crée la situation coloniale chez les colonisés comme chez les colonisateurs. Le travail de Franz Fanon ${ }^{29}$ est évoqué dans un nouveau paragraphe inséré en 1963 mais pour en souligner surtout le caractère « violent et lyrique » même si Balandier reconnaît à ces études "dues à des militants ou à des témoins du nationalisme combattant ", un éclairage sur les mutations des structures sociales en cours liées à la déco-

26 Max Gluckman, "Malinowski, Functionnal Analysis of Social Change", Africa (1947/2).

27 Georges Balandier, "La notion de situation coloniale ", op. cit., 1955, p. 31 (1963, p. 30) (cf. note 9).

28 Albert Memmi, Portait du colonisé. Portrait du colonisateur, Paris, Gallimard, 1985.

29 Franz Fanon, L'an $V$ de la Révolution algérienne, Paris, Maspero, 1959 ; Les damnés de la terre, Paris, Maspero, 1961. lonisation elle-même, y compris dans la sphère familiale et la condition des femmes ${ }^{30}$.

L'auteur préfère insister sur l'impact qu'a la décolonisation sur les anthropologues eux-mêmes, le vocabulaire utilisé jusqu'alors pour qualifier les sociétés «primitives » ou " archaïques », le doute qui s'insinue « quant à la portée de l'entreprise anthropologique » lorsque l'objet d'étude reprend l'initiative, contredit la supposée « passivité » et bouscule «le monopole de l'explication ${ }^{31}$. Ces remarques sont prémonitoires des travaux critiques ultérieurs qu'on voit se développer dans les années 1990.

Il faut remarquer que les versions successives de Balandier mobilisent une bibliographie qui dresse une géographie particulière des « situations coloniales » observées, parcourues ou simplement évoquées. L'Afrique noire francophone est privilégiée en tant que terrain de spécialité de l'auteur, avec ses anthropologues (Paul Mercier), ses praticiens (Robert Delavignette), ses historiens (Henry Brunschwig et CharlesAndré Julien), ses militants (Léopold S. Senghor). Vient ensuite l'Afrique du Sud, terrain phare des enquêtes de culture contact entre les deux guerres et des travaux critiques dans la lignée de Malinowski, dont l'organisation sociale fondée sur la ségrégation raciale est prise pour modèle de société coloniale par Balandier. Liés à cette

30 Georges Balandier, "La notion de situation coloniale ", op. cit., 1963, p. 33 (cf. note 9).

31 ld. 
question des races, les Amériques, le sud des États-Unis et le Brésil (avec Wirth et Bastide) sont mobilisés. L'espace colonial ici considéré sous l'angle sociologique des rapports sociaux et de races est plus large que l'espace colonial au sens politique du terme. Enfin, est évoquée l'Indochine française du fait de la qualité des travaux d'obédience marxiste qui y sont conduits.

Reste un grand absent, « l'Afrique française » qui offre un modèle de « situation coloniale » sur lequel Balandier aurait pu fort logiquement se pencher plutôt que de choisir la société d'Afrique du Sud, et en particulier l'Algérie. Les références que l'auteur lui consacre sont limitées. On notera que Charles-André Julien, connu surtout pour Histoire de l'Afrique du Nord (1931) ou son Afrique du Nord en marche (1952) n'est cité que pour son Histoire de l'Afrique (1944) et sa critique de l'impérialisme. Les travaux de Jacques Berque sont ignorés et ceux d'Albert Memmi aussi comme on l'a vu, ceux de Jean Dresh ou Franz Fanon rapidement évoqués. Peut-être Georges Balandier ne se sent-il pas assez familier de «la situation coloniale » en Algérie pour en parler dans le détail ou peut-être est-elle trop difficile à penser pour celui qui cherche à garder une distance critique et veut l'observer " comme un fait relevant de l'observation scientifique et indépendamment des jugements moraux qu'elle provoque $»^{32}$.

32 Ibid., p. 6.

\section{La genèse d'une notion}

La formule Contact Situation fut utilisée par Malinowski dès 1938 dans son introduction au volume Methodology of Study of Culture Contact $^{33}$ pour signifier le contexte particulier qui caractérise le " contact » entre deux sociétés et les déterminismes spécifiques qui en résultent ${ }^{34}$. Max Gluckman, dans son célèbre texte Analysis of Modern Zululand, publié en $1940^{35}$, inaugurait la notion de Social Situation pour circonscrire l'analyse intensive d'interactions dans un contexte donné tandis que Sartre reprenait le terme de situation dans sa philosophie de l'existentialisme.

La notion de " situation coloniale » a été inaugurée selon Georges Balandier par Louis Wirth dans ses travaux sur les minorités ${ }^{36}$ mais c'est Octave Mannoni dans son ouvrage Psychologie de la colonisation publié en 1950 qui en fait un premier usage réfléchi. Mannoni attaque LévyBruhl et les ethnologues qui, cherchant à comprendre la « mentalité primitive », se refusent à considérer les effets de la rencontre entre

33 Memorendum XV. International Institute of African Languages and Cultures, 1938.

34 Cf. Lucy Mair, "Malinowski and the Study of Social Change", in Raymond Firth, ed., Man and Culture. An Evaluation of the Work of Bronislaw Malinowski (New York: Routledge, 1957).

35 Max Gluckman, "Analysis of Modern Zululand", Bantou Studies (1940/14).

36 Louis Wirth, "The Present Position of Minorities in the United States", Studies in Political Science and Sociology (1941), p. 137-156; "The Problem of Minority Groups", in Ralph Linton, ed., The Science of Man in the World Crisis (New York: Columbia University Press, 1945), p. 347-372. 
« indigènes observés » et observateurs. Il est quant à lui, intéressé par les aspects psychopsychanalytiques de la relation entre colonisés et colonisateurs. " Il s'agit des phénomènes qui se manifestent dans une situation coloniale et de la manière dont, à une telle situation, réagissent les coloniaux autant que les colonisés $»^{37}$.

«Une situation coloniale est caractérisée par le fait que la différence de structure des personnalités est grande, plus grande que dans n'importe quelle autre situation [...]. L'exploitation coloniale ne se confond pas avec les autres formes d'exploitation, le racisme colonial diffère des autres formes de racisme $»^{38}$.

Mannoni est donc attentif à la spécificité des rapports que la « situation coloniale » engendre entre colonisés et colonisateurs qui, selon lui, créent des situations de malentendus et d'incompréhension. Il insiste sur la nécessité d'expliciter le terme colonial en tant qu'adjectif et non en tant que substantif. « Autrement dit, dans une situation coloniale, l'économie est coloniale ; et c'est la signification de l'adjectif, dans ce travail, non pas celle du substantif, qui nous retient. Les colonisés ont compris depuis longtemps l'importance de cet adjectif $»^{39}$. Il prend l'exemple de l'Européen colonial que les colonisés savent très bien distinguer de l'Européen.

37 Octave Mannoni, Le racisme revisité, Madagascar, 1947, Paris, Denoël, 1997 (1 ${ }^{\text {re }}$ éd. 1950), p. 55.

38 Ibid., p. 61.

39 Ibid., p. 69.
« Ils ont eu assez l'occasion de voir la transformation s'opérer sous leurs yeux. Par exemple, les Malgaches qui habitent actuellement la France sont au mieux avec les Français. Mais ils évitent avec soin la plupart des Français qui ont fait quelque séjour à Madagascar. Ainsi un "indigène" européanisé peut vivre en milieu européen sans que se crée nécessairement une "situation" particulière $»^{40}$.

Mannoni amorce ainsi une réflexion sur la fabrication conjointe du colonisateur et du colonisé à travers l'analyse des projections réciproques, des interdépendances et des interrelations; réflexion qui sera poursuivie et approfondie par la suite par Franz Fanon et Albert Memmi. « Les phénomènes d'interpsychologie » qui intéressent en tout premier lieu Mannoni ont stimulé l'attention de Balandier. Celui-ci rédige un compte rendu de l'ouvrage dans le volume IX des Cahiers internationaux de sociologie (1950). L'approche lui semble riche mais partielle car limitée aux aspects psychologiques d'un rapport colonial qu'il entend étudier, en tant que sociologue, comme une totalité.

Il rappelle que la notion de "situation coloniale » doit être rapprochée du concept de " conjoncture sociale particulière » de Georges Gurvitch ou encore du "phénomène social total » de Marcel Mauss et comprise dans son ensemble et en tant que système ${ }^{41}$. Jusqu'alors, les sciences sociales avaient largement occulté

40 ld.

41 Georges Balandier, « La situation coloniale. Approche théorique », op. cit., 1951, p. $76-77$ (cf. note 2). 
l'analyse de cette "situation coloniale ». Et Balandier attaque en tout premier lieu sa propre discipline, l'anthropologie, dont on sait que la construction même dans la seconde moitié du $\mathrm{XIX}^{\mathrm{e}}$ siècle et première moitié $\mathrm{du} \mathrm{XX}^{\mathrm{e}}$ siècle $\mathrm{a}$ consisté à abstraire l'analyse du contexte social et historique immédiat dans lequel se trouvaient les peuples étudiés. Qu'il s'agisse des évolutionnistes et diffusionnistes " avides », selon Balandier, « de spéculations théoriques méditant sur le destin des civilisations ou les origines de la société ${ }^{42}$, ou qu'il s'agisse des théoriciens de la culture « obsédés par la poursuite de l'ethnologiquement pur, du fait inaltéré et conservé miraculeusement ${ }^{43}$, tous ont de fait soigneusement refusé de penser la « situation » dans laquelle se trouvaient les communautés qu'ils observaient au moment même où ils les étudiaient, refusant par là de penser les effets de la colonisation, qu'ils n'ignoraient pourtant pas. Balandier dénonce cette posture anhistorique qui caractérise l'anthropologie de son temps ${ }^{44}$ mais fait une place particulière à l'école de Malinowski dont l'analyse a connu une inflexion notable dans les années

42 lbid., p. 45.

$43 \mathrm{ld}$.

44 II ignore les efforts d'Evans Pritchard qui, pourtant, énonce en 1950 dans une conférence donnée à Oxford à la mémoire de l'anthropologue Marett et dans un texte publié la même année dans Man, un appel en faveur d'une anthropologie capable de rendre compte de faits historiques. Mais Evans Pritchard reste surtout préoccupé du « point de vue africain » et de l'histoire proprement africaine en occultant l'intrusion européenne et ses effets qui sont, de fait, exclus de l'analyse.
1920 comme l'ont montré d'excellents travaux qui lui ont été consacrés ${ }^{45}$. Déplaçant ses terrains d'investigation du Pacifique vers l'Afrique, le maître de la monographie fonctionnaliste jusque-là entièrement consacrée à l'étude d'un système synchronique "inaltéré », se tourne vers l'analyse du « changement culturel » ou Culture Change. Il se rapproche de l'Institut international des langues et cultures africaines créé en 1926 dont la fonction est de soutenir l'action de l'administration coloniale et c'est dans ce cadre qu'il propose de construire une anthropologie pratique, soucieuse de prendre en compte la modernité du temps, à savoir les effets de colonisation. Au cœur des enjeux, « l'indigène en mutation » qu'il convient de comprendre et d'accompagner dans le processus de changement qu'il subit. Max Gluckman d'abord, puis Georges Balandier ensuite, firent, on le sait, une critique sévère de la méthodologie de Culture Change que développa Malinowski dans les années 1930. Mais notons que celuici fut un des premiers, en anthropologie, à tenter de penser ce qu'on pourrait appeler les " effets de contexte " à savoir la colonisation et son impact sur les sociétés locales et en s'interrogeant par là même sur la ques-

45 Georges W. Stocking, Functionnalism Historicized. Essays on British Social Anthropology (Madison: University of Wisconsin Press, 1984); Colonial Situations. Essays on the contextualization of ethnographic knowledge (Madison: University of Wisconsin Press, 1991); Benoît de l'Estoile, "L'Afrique comme laboratoire. Expériences réformatrices et révolution anthropologique dans l'empire colonial britannique (1920-1950) ", thèse de doctorat sous la direction de Christian Topalov, EHESS, Paris, 2004. 
tion de l'histoire, de l'usage du passé et de la mémoire. Poursuivant dans les années 1910, un certain romantisme primitiviste à l'image de ses maîtres, il voit d'abord dans les îles Trobriand, proches de la Papouasie NouvelleGuinée, l'exemple même de ces espaces où l'influence de l'homme blanc est encore dérisoire, voire inconnue.

À y regarder de plus près, cependant, ce n'est déjà plus le cas et Malinowski ne l'ignore pas, comme il en témoigne en 1935 devant ses étudiants :

«Les faits empiriques que l'ethnographe a devant lui aux Trobriand aujourd'hui, ne sont pas des indigènes non touchés par l'influence européenne mais des indigènes qui, dans une large mesure, ont été transformés par cette influence. Le Trobriandais tel qu'il était il y a une génération ou deux est devenu une chose du passé qui peut être reconstruit mais non observé $»^{46}$.

La remarque est cruciale et témoigne d'une modification en profondeur du point de vue anthropologique, y compris au sujet de ces zones lointaines et mythiques du Pacifique Sud que les Européens considèrent comme une Last Frontier. Plutôt que de ne retenir que le «traditionnel » en se détournant de ce qui l'altère comme peuvent le faire au même moment Griaule ou Leiris, Malinowski et ses élèves, à partir de terrains situés dans les zones

46 Benoît de l'Estoile, "L'Afrique comme laboratoire », op. cit., p. 727 (cf. note 45). de l'Afrique australe les plus lourdement marquées par les multiples effets de la colonisation, cherchent en tâtonnant à saisir la réalité qu'ils observent. Pour autant Malinowski récuse les efforts d'historicisation auxquels ses élèves comme Lucy Mair et Monica Hunter se livrent. "Il serait vain, dans un but de reconstitution, de faire confiance aux souvenirs de vieillards ou aux comptes rendus du passé ${ }^{47}$. Le manque de sources dans le cas de l'histoire africaine ou encore leur caractère fragmentaire ne permet pas de "procéder par déductions irréfutables, c'est-à-dire sur la base de généralisations scientifiques $»^{48}$. Quant aux archives, elles ne sont qu'un «Paper Program qui ne reflète jamais l'actualité du contact ${ }^{49}$. Malinowski préfère s'intéresser à l'observation de la situation présente, à « la réalité de ce qui survit comme résidu vital d'un passé historique ${ }^{50}$.

Pour Gluckman, Malinowksi refuse de « distinguer la compréhension d'une culture par le biais de la connaissance de son histoire et l'analyse des processus historiques à l'œuvre et la connaissance qu'en ont les membres de cette culture ainsi que la signification qu'ils

47 Bronislaw Malinowski, The Dynamics of Culture Change (New Haven: Yale University Press, 1945), p. 56.

48 Ibid., p. 61.

49 Max Gluckman, "Malinowski Functionnal Analysis of Social Change", op. cit., p. 104 (cf. note 26).

50 Bronislaw Malinowski, The Dynamics of Culture Change, op. cit., 1945, p. 67 (cf. note 47). 
en donnent $»^{51}$. La contradiction de sa méthodologie est d'autant plus forte qu'il prétend éclairer le « changement » en excluant l'analyse des processus historiques et la genèse des phénomènes. D'où la faiblesse de certaines de ses observations que Gluckman et Balandier soulignent aisément. Malinowski affirme que les guerres intertribales ont disparu en Afrique avec l'occupation européenne et font ainsi partie d'un passé mort et révolu, et Gluckman de lui répondre que seule l'expression militaire des conflits a disparu mais que les hostilités se sont déplacées dans d'autres registres d'action $^{52}$. De la même manière, la disparition du cannibalisme ou celle des mises en esclavage au sein des mondes africains ne signifie pas un oubli ou une perte de sens de ces pratiques mais un déplacement sous d'autres formes. Le plus problématique dans la posture de Malinowski est sans aucun doute son refus de prendre en compte l'histoire du contexte colonial et ses acteurs, y compris les Européens, saisis alors comme sujets d'enquête au même titre que les Indigènes, comme le suggèrent deux de ses meilleurs étudiants, Meyer Fortes et Isaac Shapira. Ce refus témoigne d'un aveuglement pour une situation coloniale construite comme totalité sociologique par-delà les conflits et les ségrégations qui la caractérisent et que Maloniwski veut ignorer. D'où cette vision partielle qui consiste à ne privilégier que l'étude

51 Max Gluckman, "Malinowski Functionnal Analysis of Social Change”, op. cit., p. 104 (cf. note 26).

52 ld. des mondes africains et les changements qu'ils subissent sans porter attention aux politiques et pratiques européennes qui s'imposent à eux.

\section{Situation coloniale, fragmentation et hiérarchisation}

Il faut noter que Balandier se garde de donner $a$ priori une définition de ce qu'il appelle dans son introduction « ce complexe qualifié de situation coloniale ». Il se contente de poser le parti pris de la totalité en début de texte comme signe distinctif d'une approche qui trancherait dans les sciences sociales avec le caractère partiel des études menées jusque-là. Il ne se risque finalement à une ébauche de définition qu'en conclusion de son article en procédant d'abord à une énumération, qui est maintenue dans chacune de ses versions successives moyennant quelques corrections entre celle de 1951, ici reprise, et celle de 1963 :

" La domination imposée par une minorité étrangère, racialement (ou ethniquement) ${ }^{53}$ et culturellement différente au nom d'une supériorité raciale (ou ethnique) et culturelle dogmatiquement affirmée, à une majorité autochtone matériellement inférieure ; cette domination entraîne la mise en rapport de civilisations radicalement hétérogènes : une civilisation à machinisme, à économie puissante, à rythme rapide et d'ori-

53 Le « racialement (ou ethniquement) » est simplifié dans la version de 1963 avec la formule « racialement » entre guillemets. 
gine chrétienne, s'imposant à des civilisations sans technique complexe, à économie arriéré $e^{54}$, à rythme lent, et radicalement non chrétienne ; le caractère fondamentalement antagoniste des relations existant entre ces deux sociétés qui s'explique par le rôle d'instrument auquel est condamnée la société colonisée ; la nécessité, pour maintenir la domination de recourir non seulement à la "force" mais encore à un système de pseudo-justifications et de comportements stéréotypés, etc. $»^{55}$.

On note que la « situation coloniale » est ici essentiellement saisie sous l'angle de la relation de domination qui s'exerce entre deux sociétés radicalement hétérogènes et antagonistes par la force (que Balandier met entre guillemets comme pour en atténuer le sens) et par l'idéologie. Mais cette énumération ne satisfait pas l'auteur car "à la faveur des "vues" particulières prises par chacun des spécialistes » et sur la base du modèle sociologique qu'il tente de construire, la situation coloniale doit être saisie en tant que système liant ensembles des éléments formant totalité. En 1951, l'auteur s'emploie à la définition de cette totalité :

"Cette totalité met en cause les "groupements" composant "la société globale" (la colonie) comme les représentations collectives propres à chacun de ceux-ci ; elle se sait à tous les niveaux de la réalité sociale. Mais en raison même du caractère hétérogène des groupements, des

54 Devient « une économie retardée » en 1963.

55 Georges Balandier, «La situation coloniale. Approche théorique », op. cit., 1951, p. 75 (cf. note 2). "modèles" culturels, des représentations mises en contact, des transformations apportées au système chargé de maintenir artificiellement les rapports de domination et de soumission, la situation coloniale se modifie profondément et à un rythme rapide $»^{56}$.

En 1963, la définition de la totalité est réduite aux groupements composant " la société globale » (la colonie) ainsi qu'aux « représentations collectives propres à chacun d'entre eux $»^{57}$.

C'est à Eric Anderson Walker, historien britannique et sud-africain de renom entre les deux guerres, auteur de plusieurs tomes de la célèbre Cambridge History of the British Empire, que Balandier emprunte la formule "société globale » pour désigner un ensemble difficile à nommer tant il oppose des « groupes » (selon Walker) ou « groupements » (selon Balandier) «plus ou moins conscients de leur existence, souvent opposés les uns aux autres par leur couleur et qui s'efforcent de mener des vies différentes dans un cadre politique unique $»^{58}$. Ces « groupes » ou « groupements » ont pour caractéristique première de présenter une hétérogénéité radicale :

« [Ils] parlent des langues différentes, ont une nourriture différente, se livrent souvent à des occupations différentes qui leur sont désignées

56 Ibid., p. 76.

57 Georges Balandier, "La notion de situation coloniale ", op. cit., 1963, p. 35 (cf. note 9).

58 Ibid., p. 15 
par la loi ou la coutume, portent des vêtements différents [...], vivent dans des types différents d'habitations, chérissent des traditions différentes, adorent des dieux différents, entretiennent des idées différentes du bien et du mal $»^{59}$.

Rien ici qui ressemble à ce que la sociologie du temps appelle « une communauté » fondée sur un système de solidarité, le partage de valeurs communes et d'objectifs communs. Retenons ici l'idée de la fragmentation particulière qui caractériserait les « sociétés globales » en situation coloniale comme l'une des pistes suivies par les analyses les plus récentes des univers coloniaux ; on pense en particulier à l'école des Subaltern Studies qui a particulièrement insisté sur la diversité des systèmes de pensées, de valeurs et de pratiques que recouvraient l'Inde coloniale et l'exercice d'un pouvoir sans hégémonie au sens gramscien du terme, celle d'un consensus négocié entre groupes dominants et dominés ${ }^{60}$.

À cette idée de fragmentation s'ajoute celle d'un déséquilibre constitutif des « sociétés globales » entre une minorité numérique d'origine européenne agissant comme une majorité sociologique sur une majorité autochtone réduite à une minorité sociologique. «Cette minorité européenne agit sur les peuples autochtones avec une vigueur disproportionnée au nombre ; elle est, si l'on veut, extrêmement contagieuse,

59 lbid., p. 16.

60 Ranajit Guha, "Dominance without Hegemony and its Historiography", Subaltern Studies (1989/VI). et de sa nature déformante $»^{61}$. Car l'usage du concept de " minorité », dans la lignée des travaux de l'école de Chicago et de Louis Wirth en particulier, renvoie à des liens de domination et de subordination et non à des questions d'ordre statistique. Et Balandier, avec finesse, souligne le renversement des perceptions en cours en Afrique du Sud dans ces années cinquante lorsque

« la population blanche commence à voir dans sa situation un problème de minorité, alors que les Noirs voient dans la leur un problème colonial et de tutelle; ainsi, en Algérie où la minorité européenne a défendu âprement son statut ${ }^{62}$.

La situation coloniale recouvre une société hétérogène dominée par une minorité agissante qui organise une hiérarchie des " groupes » sur le principe fondamental de la « race ». Maunier plaçait au cœur de sa sociologie coloniale «le contact des races » et Balandier voit dans la race un élément structurant essentiel puisqu'il s'appuie sur l'exemple de l'Afrique du Sud et plus largement les exemples de sociétés fortement marquées par la racialisation des rapports sociaux et l'existence de ce qu'on appelle la Colour Line ou Colour Bar qui trouve une réalité pratique dans les politiques publiques de ségrégation. La carte mentale implicite que Balandier mobilise à ce sujet dépasse les territoires coloniaux au sens strict et fait bouger ici les frontières de la notion de « situation coloniale » qui peut recou-

61 Georges Balandier, "La notion de situation coloniale ", op. cit., 1963, p. 16 (cf. note 9).

62 Id. 
vrir sous cet angle le sud des États-Unis ou les territoires d'Amérique latine que décrit Roger Bastide, évoquant ainsi l'idée intéressante que sociologie coloniale et pratiques coloniales ne recouvrent pas exactement la carte politique coloniale ou encore que des États indépendants et souverains peuvent connaître de fait certains aspects de la situation coloniale.

La hiérarchisation à l'œuvre dans la situation coloniale repose aussi sur la civilisation selon la lecture évolutionniste classique : « Le primitif et le civilisé, le païen et le chrétien, les civilisations techniques et les civilisations "arriérées" ${ }^{63}$. À ces principes structurants, Balandier ajoute celui de la nationalité. Il distingue en effet le cas spécifique des étrangers de race blanche et semble ici se référer spécifiquement à l'exemple de l'Afrique occidentale française (AOF) et au groupe des Libanos-Syriens.

« Ceux-ci constituent une minorité au sens plein du terme, numériquement et sociologiquement ; ils peuvent avoir un statutéconomique important, ils n'en sont pas moins soumis à un contrôle administratif. Ils sont suspects en raison de leur nationalité et sont souvent coupés de la "véritable" société coloniale ${ }^{64}$.

De fait, il conviendrait de réfléchir sur les similitudes que l'on peut trouver ici avec le traitement réservé à d'autres " étrangers de race blanche », les Italiens en Tunisie, les Espagnols ou Maltais en Algérie, les Levantins au Maghreb

63 Ibid., p. 22.

64 Ibid., p. 19. jusqu'aux Irlandais en Australie ; minorité que l'on accuse dans certains cas d'une plus grande familiarité ou intimité avec la société autochtone (comme c'est le cas semble-t-il des LibanoSyriens en AOF) à l'instar d'ailleurs d'autres groupes intermédiaires "étrangers de couleur » comme les Indiens en Afrique du Sud, ou encore les Japonais en Nouvelle-Calédonie ou les Chinois en Indochine ${ }^{65}$. Ce faisant, Balandier nous invite à prêter une attention soutenue aux nuances des hiérarchies coloniales, à la logique des catégorisations et identifications. Dans cette attention qu'il porte aux groupes intermédiaires et à leur position, Balandier évoque la question des métis qu'il voit condamnés à l'isolement car incarnant un « compromis racial » inclassable ou dérangeant dans l'ordre de la hiérarchie coloniale ; métis invisibles à l'exception du cas des Bastards de Rehoboth dans l'ancien Sud-Ouest africain allemand qui cherchèrent à s'unifier. S'ils incarnent un compromis racial, ils ne peuvent personnifier un compromis social, du moins en Afrique du Sud où ils furent rejetés vers une société noire auxquels eux-mêmes refusaient d'être assimilés et échouèrent à construire une unité « non européenne » avec les Indiens et les Bantous en 1927. La question métisse est plus complexe que cela et varie considérablement selon les situations coloniales observées. On rappellera la condition privilégiée des « Demis » en Polynésie française ou encore la réussite économique et politique de certaines grandes familles métisses

$65 I d$. 
en Nouvelle-Zélande. On rappellera aussi la complexité des logiques juridiques qui furent mises en œuvre dans l'empire français dans l'entredeux-guerres pour intégrer les enfants métis de père inconnu, et en tout premier lieu les enfants eurasiens de l'Indochine française dans la citoyenneté française ${ }^{66}$. Il y a là un point plus général que Balandier ne semble pas avoir envisagé. Si la race, « l'état de civilisation » et plus marginalement la nationalité comptent comme critères de hiérarchisation des groupes en situation coloniale, le statut juridique des uns et des autres est peut être le plus opérant de tous. Dans l'empire français, c'est moins la nationalité qui ordonne les groupes que le clivage fondamental entre « citoyens » et « sujets », auquel s'ajoute pour ces derniers la possibilité d'appliquer un régime d'exception : le régime de l'indigénat. Le droit, au carrefour de la citoyenneté, de la nationalité et de la race, ordonne les univers sociaux coloniaux et produit un intense travail d'identification et de catégorisation pour tenter de stabiliser de nouvelles frontières entre les groupes et de reclasser les individus aux marges. La piste a été pressentie par Balandier et s'avère aujourd'hui féconde ${ }^{67}$.

66 Emmanuelle Saada, Les enfants de la colonie. Les métis dans l'Empire français. Entre sujétion et citoyenneté, Paris, La Découverte, 2007 ; Yerri Urban, « Race et nationalité dans le droit colonial français ", thèse de doctorat sous la direction de Patrick Charlot et Patrick Weil, Université de Bourgogne, 2009.

67 Voir les travaux de Laure Blévis ou de Frederick Cooper et Jane Burbank pour ne citer que ces auteurs.

\section{Puissance, inauthenticité et ambiguïtés}

Au sommet de la « société globale », une minorité « déformante » agissant sur les peuples autochtones «de façon disproportionnée ${ }^{68}$. Et Balandier de s'appuyer sur l'ouvrage de Robert Delavignette qui fut gouverneur de l'AOF, espace colonial caractérisé par la prépondérance de l'administration et le faible nombre de colons, pour nous décrire une société coloniale « de provenance et d'attache métropolitaine [...] à caractère bourgeois animée par la notion de supériorité héroïque » et « l'esprit féodal », composée surtout d'hommes jeunes dans les «colonies dites d'encadrement " ${ }^{69}$. Notons ici l'allusion qui est faite à la contradiction entre l'origine bourgeoise d'hommes issus des élites européennes de la fin du XIX ${ }^{\mathrm{e}}$ siècle et les valeurs « héroïques » ou « féodales » qu'ils défendent en colonies tirées d'un référentiel de l'Ancien Régime. Ces hommes estiment appartenir à une véritable caste, soucieuse de maintenir ses distances et d'éviter toute familiarité avec ceux qui n'en sont pas. Représentants de la France et de la race française, ils défendent la distinction, le prestige et les privilèges attachés à leur statut et leur rang. La « situation coloniale » favoriserait ainsi la remobilisation de normes et de comportements légitimés par des logiques d'honneur et des logiques de cours aristocratiques que ces élites républicaines en colonies endossent, alors même qu'elles ont été éduquées par un autre

68 Georges Balandier, "La notion de situation coloniale», op. cit. 1963, p. 16 (cf. note 9).

69 Ibid., p. 18. 
système de valeurs fondé sur l'idée du pouvoir rationnel bureaucratique. Balandier lance ainsi l'idée que les terrains coloniaux peuvent être, en quelque sorte, des espaces de restauration de références puisées dans un vocabulaire politique ancien. Il s'appuie ici sur les valeurs, les pratiques et les discours de ce qu'il appelle « les coloniaux prépondérants $»^{70}$; l'enjeu étant de défendre l'étiquette du conquérant autant que d'imposer au conquis le respect de celui-ci dans les faits comme dans le geste. Cette restauration de normes tirées de l'Ancien régime se joue aussi ailleurs, dans la réorganisation de certaines hiérarchies indigènes à travers le prisme de l'organisation féodale, au prétexte d'une similitude avec « l'organisation traditionnelle ».

L'idée reste à creuser mais il ne s'agirait pas d'exagérer la portée de ce modèle « héroïque » d'un fonctionnaire colonial soucieux de défendre «la dignité » et le «prestige »; il s'agit d'abord d'un modèle performatif plus ou moins respecté et dont la fonction va surtout servir à tenter de réguler les relations entre les représentants de la puissance coloniale et les indigènes jusque dans leurs aspects les plus intimes, comme l'ont montré les travaux portant sur les mariages, le traitement des enfants métis ou la condamnation des comportements déviants de "pauvres Blancs » enclins à une trop grande familiarité avec les mondes indigènes. Loin de toute

70 Emmanuelle Saada, "The Empire of Law, Prestige, and Domination in 'the Colonial Situation"', French Politics, Culture and Society, vol. 20 (2002). réification d'une catégorie du «colonial», Balandier note que la société coloniale est profondément hétérogène, divisée en factions aux intérêts divergents et occupant des positions différentes. On ne peut que souligner l'importance de cette remarque qui est un appel à une sociologie fine des différentes composantes de cette société coloniale, des divisions et des contradictions qui la traversent. Cette société coloniale, pourtant, aussi divisée ou inégalitaire qu'elle puisse être, apprend à partager un sentiment commun, la supériorité de la race blanche. Pour Balandier, la situation coloniale se présente comme saturée par l'idéologie et les stéréotypes renvoyés par les représentants de la nation conquérante sur le reste de la population et en tout premier lieu la société colonisée. À la conviction de la supériorité de la race blanche s'ajoute la disqualification de l'Autre: l'incapacité des indigènes à se diriger, l'archaïsme des pouvoirs politiques traditionnels ou le despotisme des chefs, les tendances forcément dictatoriales des nouveaux leaders ou encore, dans le registre économique, l'incapacité à mettre en valeur les ressources, la paresse, l'évidence d'une pauvreté à venir en cas de départ de la nation conquérante, celle-ci étant vue comme le moteur de toute modernité. L'ensemble de cet argumentaire bien connu constitue un appareil de justifications dont le but est de confirmer la supériorité des uns et l'infériorité des autres en créant un écran de représentations entre les uns et les autres. D’où l'effet de déréalisation 
que produit cet ensemble idéologique chez l'Européen colonial, qui l'empêche d'envisager le colonisé autrement et marque profondément les rapports sociaux. L'intérêt dans ce qu'avance ici Balandier n'est pas de s'en tenir au seul registre des représentations mais de comprendre le sens et l'effet de cette production idéologique pour la construction de l'individu colonial. Balandier introduit les failles de ce qu'il appelle une «doctrine incertaine ». L'appareil de justification n'est pour lui qu'un habillage d'une réalité plus brutale, l'exploitation pure et simple et le principe de la colonisation comme fait de puissance au profit des membres de la société coloniale d'abord, au profit de la métropole ensuite. L'historien Henri Brunschwig appelait malentendus ce que Louis Joubert qualifiait de pure hypocrisie, c'est-à-dire le décalage entre l'exercice de la domination et l'ordre des représentations et des justifications. L'écart entre pratiques et croyances est pour Balandier une des caractéristiques essentielles de la situation coloniale: son inauthenticité, liée aux mensonges essentiels qu'elle comporte, qui habillent les faits bruts et dont les acteurs ont conscience. Albert Memmi reprendra l'idée en décrivant le système colonial comme un système de privilège fondamentalement illégitime dont profitent les plus puissants comme les plus humbles des Européens, et ce comparativement et au détriment des colonisés. L'illégitimité de la situation coloniale fait de l'Européen aux colonies un usurpateur qui ne peut défendre sa position qu'au prix d'un intense travail de justification fondée sur la supériorité de sa race ${ }^{71}$. La relation coloniale, pour l'ensemble de ces auteurs, fabrique autant le colonial que le colonisé, forgeant un couple dont le lien réciproque est pathologique.

Le texte de Georges Balandier, rédigé une première fois en 1951, introduit à partir de 1955 son ouvrage phare Sociologie actuelle de l'Afrique noire, consacré à l'analyse sur le long terme des effets de la situation coloniale sur les sociétés Fang et Ba-Kongo. Comme Malinowski et Gluckman, Balandier est d'abord fasciné par l'étude des changements au sein des sociétés africaines. S'il ne s'agit plus de réduire l'angle d'observation au seul " indigène en mutation », celui-ci reste cependant l'objet primordial de l'enquête. La notion de situation coloniale apparaît alors comme une introduction générale d'un programme qui se concentre par la suite sur " l'étude diachronique et relationnelle des sociétés dites hier "primitives" », comme l'indique la conclusion modifiée de la version de 1963, au service d'une anthropologie et sociologie dynamique. On doit ici noter le décalage qui apparaît entre la lecture que fait Balandier de la société colonisée en 1951 puis dans son premier chapitre de Sociologie actuelle, assez lourdement marquée par le rapport dominant/dominé, et la finesse des analyses dont témoignent l'ouvrage en son entier et l'œuvre qui le suivra.

71 Albert Memmi, Portait du colonisé. Portrait du colonisateur, Paris, Gallimard, 1985, p. 34. 
La société colonisée apparaît dans « La notion de situation coloniale » comme radicalement instrumentalisée par la domination européenne, écrasée du fait de «l'absence de technique avancée, de puissance matérielle autre que celle du nombre ${ }^{72}$, du fait aussi des divisions internes et anciennes que l'intrusion de la société coloniale intensifie et démultiplie. L'importation de nouvelles religions chrétiennes crée «la confusion religieuse ", l'instauration d'un nouvel ordre politique impose de nouvelles frontières et divisions territoriales, l'exploitation économique provoque les spoliations foncières, le déplacement des populations, les réquisitions des hommes sur les chantiers ou plantations et pour finir l'exode rural et l'émergence de nouveaux « urbains ». Balandier utilise un vocabulaire clinique pour caractériser les effets de l'emprise coloniale sur les sociétés colonisées. Il rappelle les propos d'un historien qui voit dans la colonisation une véritable "chirurgie sociale » et insiste sur «l'état de crise latente» dans lequel se trouvent les sociétés assujetties qualifié de " sociopathologie ». La colonisation provoque des « désajustements » collectifs et individuels, l'altération ou la disparition d'institutions anciennes d'une part, le sentiment d'insécurité, le complexe de dépendance, l'anxiété, la névrose, la psychose d'autre part, thèmes abondamment traités par les psychologues de la colonisation comme Mannoni et surtout Franz Fanon dans les Damnés de la terre. La lecture psychologisante à laquelle semble adhérer le jeune Balandier peut surprendre aujourd'hui surtout lorsque celui-ci l'utilise en 1963 pour traiter des mouvements de luttes nationalistes :

\begin{abstract}
« Divers travaux (en général les plus polémiques [référence probable à Fanon]) ont insisté [...] sur l'état de crise que révèlent la plupart des sociétés colonisées. Ce qui est vrai dans la mesure où la minorité dominante s'oppose aux véritables solutions ; car il apparaît bien que, dans le cas de la société colonisée, la recherche de ses normes modernes coïncide avec la recherche de son autonomie. Ce fait impose au sociologue une méthode d'analyse en quelque sorte clinique ${ }^{73}$.
\end{abstract}

On est loin d'une lecture politique du moment historique observé car l'auteur est tout entier consacré à la construction de son objet sociologique ; il voit dans la crise latente et permanente que connaissent les sociétés colonisées un véritable révélateur des structures et comportements fondamentaux, des points de résistances ou des conduites novatrices. En d'autres termes, les destructions et altérations provoquées par le processus de colonisation et de modernisation constitueraient une sorte d'expérience sociologique " grossière » selon l'auteur, donnant à voir à la fois les fondements des sociétés anciennes et leurs capacités de réaction ou d'adaptation.

73 Ibid., p. 27. En italique dans le texte.
72 Georges Balandier, "La notion de situation coloniale ", op. cit., 1963, p. 20 (cf. note 9). 
L'analyse est encore approximative et signale le caractère balbutiant des recherches menées alors dans les territoires colonisés. Mais des pistes innovantes sont signalées et introduisent l'effort entrepris dans la suite de Sociologie de l'Afrique noire visant à décrire le plus finement possible les dynamiques propres des sociétés colonisées. Contre l'idée alors communément répandue d'une disparition programmée des cultures indigènes à l'épreuve de la colonisation, de la modernisation et de la mondialisation (hormis quelques enclaves préservées où subsisteraient des " sociétés primitives » aux cycles immémoriaux), la jeune génération d'anthropologues à laquelle Balandier appartient s'emploie à décrire les subtilités du « changement », la persistance de formes de sociabilité anciennes, la troublante ambiguïté des nouvelles Églises, messianismes ou prophétismes puisant dans des référentiels à la fois traditionnels et modernes, auquel le jeune Balandier s'intéresse particulièrement, ou encore les positions intercalaires qu'évoque Max Gluckman et qui confèrent à certains individus une pluriappartenance (celle par exemple d'un chef administratif qui est aussi chef traditionnel et ouvrier à la mine, celle d'un "sujet » qui prend du pouvoir par la voie du travail salarié et du syndicalisme). Contre l'idéologie coloniale disqualifiante, Balandier note aussi l'émergence de mouvements de contre-acculturation, qu'Albert Memmi appelle « contre-mythologie », qui consistent à opérer un retour sur soi dans la quête des coutumes anciennes, de la religion première, des traditions précoloniales.

Les pistes de recherche sont ouvertes et se déploieront dans l'œuvre ultérieure mais notons que la piste centrale de l'article l'analyse de la situation coloniale en soi et pour soi - quoique invoquée comme essentielle sera finalement abandonnée. La passion de l'Autre, la volonté d'accompagner les jeunes pays émergents, l'intérêt pour les grandes questions du moment, le tiers-monde, le développement, les révolutions agraires, urbaines ou encore le retour à l'histoire " précoloniale » et aux prémisses des nationalismes l'emportent largement sur la thématique coloniale désormais obsolète et dont on ne veut plus se souvenir. Dans le milieu anthropologique de l'époque, comme le rappelle George Balandier en 1999, son texte sur la « situation coloniale » fut qualifié [d'] « insensé et suicidaire ${ }^{74}$.

Ce texte suscite indéniablement aujourd'hui un regain d'intérêt, d'abord parce qu'il permet de revenir sur une littérature scientifique des années 1930, 1940, 1950 dont l'immense mérite est de témoigner d'une " situation coloniale » encore en acte, analysée par les auteurs du temps, tant au niveau des pratiques que des représentations, avec une distance critique qui fait alors rupture. Cette littérature fournit aujourd'hui un matériel historique sur lequel il conviendrait de revenir en profondeur. « La

74 «Les mondes de la surmodernité. Entretien avec Georges Balandier », Mars, n 10, 1999/10, p. 38. 
notion de situation coloniale » apporte ensuite les éléments d'une posture épistémologique inscrite dans la tradition des sciences sociales contre les facilités de l'empirisme non réflexif de l'histoire coloniale classique et les théorisations décontextualisantes des Postcolonial Studies. Cette troisième voie permet de rouvrir à nouveaux frais la boîte noire de la « situation coloniale », comme objet historique cette fois. Elle permet aussi de revenir sur les effets de la relation colonisateur/colonisé et les effets de système et de représentations que l'on considère trop souvent comme connus. La réflexion remise sur le métier de l'historien ouvre au contraire de nouvelles pistes pour mieux comprendre les formes et la nature des relations coloniales passées qui ont «fait société » en leurs temps et dont les héritages, plus ou moins occultés, pèsent encore lourd dans notre monde contemporain. 